\title{
MUTUAL EXISTENCE OF PRODUCT INTEGRALS IN NORMED RINGS
}

\author{
BY \\ JON C. HELTON ${ }^{(1)}$
}

ABSTRACT. Definitions and integrals are of the subdivision-refinement type, and functions are from $R \times R$ to $N$, where $R$ denotes the set of real numbers and $N$ denotes a ring which has a multiplicative identity element represented by 1 and a norm $|\cdot|$ with respect to which $N$ is complete and $|1|=1$. If $G$ is a function from $R \times R$ to $N$, then $G \in O M^{*}$ on $[a, b]$ only if (i) $\mathrm{n}^{y}(1+G)$ exists for $a \leq x<y \leq b$ and (ii) if $\epsilon>0$, then there exists a subdivision $D$ of $[a, b]$ such that, if $\left\{x_{i}\right\}_{i=0}^{n}$ is a refinement of $D$ and $0 \leq$ $p<q \leq n$, then

$$
\left|x_{p} \Pi^{x} q(1+G)-\prod_{i=p+1}^{q}\left(1+G_{i}\right)\right|<\epsilon ;
$$

and $G \in O M^{\circ}$ on $[a, b]$ only if (i) $\Pi^{y}(1+G)$ exists for $a \leq x<y \leq b$ and (ii) the integral $\int_{a}^{b}|1+G-\Pi(1+G)|$ exists and is zero. Further, $G \in O P^{\circ}$ on $[a, b]$ only if there exist a-subdivision $D$ of $[a, b]$ and a number $B$ such that, if $\left\{x_{i}\right\}_{i=0}^{n}$ is a refinement of $D$ and $0<p \leq q \leq n$, then $\left|\Pi_{i=p}^{q}\left(1+G_{i}\right)\right|<B$.

If $F$ and $G$ are functions from $R \times R$ to $N, F \in O P^{\circ}$ on $[a, b]$, each of $\lim _{x, y \rightarrow p^{+}} F(x, y)$ and $\lim _{x, y \rightarrow p^{-}} F(x, y)$ exists and is zero for $p \in[a, b]$, each of $\lim _{x \rightarrow p^{+}} F(p, x), \lim _{x \rightarrow p^{-}} F(x, p), \lim _{x \rightarrow p^{+}} G(p, x)$ and $\lim _{x \rightarrow p^{-}} G(x, p)$ exists for $p \in[a, b]$, and $G$ has bounded variation on $[a, b]$, then any two of the following statements imply the other:
(1) $F+G \in O M^{*}$ on $[a, b]$,
(2) $F \in O M^{*}$ on $[a, b]$, and
(3) $G \in O M^{*}$ on $[a, b]$.

In addition, with the same restrictions on $F$ and $G$, any two of the following statements imply the other:
(1) $F+G \in O M^{\circ}$ on $[a, b]$,
(2) $F \in O M^{\circ}$ on $[a, b]$, and (3) $G \in O M^{\circ}$ on $[a, b]$.

The results in this paper generalize a theorem contained in a previous paper by the author [Proc. Amer. Math. Soc. 42 (1974), 96-1031. Additional background on product integration can be obtained from a paper by $B$. W.

Helton [Pacific J. Math. 16 (1966), 297-322].

Presented to the Society, January 23, 1975; received by the editors October 4, 1974. AMS (MOS) subject classifications (1970). Primary 28 A25, 26 A39.

Key words and phrases. Sum integral, product integral, subdivision-refinement integral, existence, interval function, normed complete ring.

(1) This research was supported in part by a grant from Arizona State University. Copyright $\odot$ 1975. American Mathematical Suciety 
All definitions are of the subdivision-refinement type, and functions are from $R \times R$ to $N$, where $R$ denotes the set of real numbers and $N$ denotes a ring which has a multiplicative identity element represented by 1 and a norm $|\cdot|$ with respect to which $N$ is complete and $|1|=1$. Functions are assumed to be defined only for elements $\{x, y\}$ of $R \times R$ such that $x<y$.

If $G$ is a function from $R \times R$ to $N$, then $\int_{a}^{b} G$ exists only if there exists an element $L$ of $N$ such that, if $\epsilon>0$, then there exists a subdivision $D$ of $[a, b]$ such that, if $\left\{x_{i}\right\}_{i=0}^{n}$ is a refinement of $D$, then $\left|L-\sum_{i=1}^{n} G_{i}\right|<\epsilon$, where $G_{i}=G\left(x_{i-1}, x_{i}\right)$. Similarly, $a^{b}(1+G)$ exists only if there exists an element $L$ of $N$ such that, if $\epsilon>0$, then there exists a subdivision $D$ of $[a, b]$ such that, if $\left\{x_{i}\right\}_{i=0}^{n}$ is a refinement of $D$, then $\left|L-\Pi_{i=1}^{n}\left(1+G_{i}\right)\right|<\epsilon$.

The statements that $G$ is bounded on $[a, b], G \in O P^{\circ}$ on $[a, b]$ and $G \epsilon$ $O B^{\circ}$ on $[a, b]$ mean there exist a subdivision $D$ of $[a, b]$ and a number $B$ such that, if $\left\{x_{i}\right\}_{i=0}^{n}$ is a refinement of $D$, then

(1) $\left|G_{i}\right|<B$ for $1 \leq i \leq n$,

(2) $\left|\Pi_{i=p}^{q}\left(1+G_{i}\right)\right|<B$ for $1 \leq p \leq q \leq n$, and

(3) $\sum_{i=1}^{n}\left|G_{i}\right|<B$,

respectively.

Let $G\left(p, p^{+}\right), G\left(p^{+}, p^{+}\right), G\left(p^{-}, p\right)$ and $G\left(p^{-}, p^{-}\right)$represent $\lim _{x \rightarrow p^{+}} G(p, x), \lim _{x, y \rightarrow p^{+}} G(x, y), \lim _{x \rightarrow p^{-}} G(x, p)$ and $\lim _{x, y \rightarrow p^{-}} G(x, y)$, respectively. Now, $G^{x} \in S_{1}$ on $[a, b]$ only if $\vec{p}^{x}\left(p^{+}, p^{+}\right)$exists and is zero for $a \leq p<b$ and $G\left(p^{-}, p^{-}\right)$exists and is zero for $a<p \leq b$; and $G \in S_{2}$ on $[a, b]$ only if $G\left(p, p^{+}\right)$exists for $a \leq p<b$ and $G\left(p^{-}, p\right)$ exists for $a<p \leq$ b. Further, $G \in O L^{\circ}$ on $[a, b]$ only if $G\left(p, p^{+}\right)$and $G\left(p^{+}, p^{+}\right)$exist for $a \leq$ $p<b$ and $G\left(p^{-}, p\right)$ and $G\left(p^{-}, p^{-}\right)$exist for $a<p \leq b$.

For additional background on product integration, the reader is referred to papers by P. R. Masani [10], J. S. MacNerney [9], B. W. Helton [2] and the author [7].

Suppose $F$ and $G$ are functions on $R \times R$. If $\int_{a}^{b} F$ exists and $\int_{a}^{b} G$ exists, then it is easily shown that $\int_{a}^{b}(F+G)$ exists. However, if $\prod_{x}^{y}(1+F)$ and $\Pi^{y}(1+G)$ exist for $a \leq x<y \leq b$, it does not necessarily follow that ${ }_{x} \Pi^{y}(1+F+G)$ exists for $a \leq x<y \leq b$. The purpose of this paper is to investigate the existence of such product integrals. In particular, with suitable restrictions on the functions involved, we interrelate the existence of $\Pi_{x}^{y}(1+F),{ }_{x} \Pi^{y}(1+G)$ and $\Pi^{y}(1+F+G)$. However, before stating our results, we need several additional definitions.

First, $G \in O A^{\circ}$ on $[a, b]$ only if $\int_{a}^{b} G$ exists and $\int_{a}^{b}\left|G-\int G\right|=0$. Second, $G \in O M^{\circ}$ on $[a, b]$ only if $\Pi^{y}(1+G)$ exists for $a \leq x<y \leq b$ and $\int_{a}^{b}|1+G-\Pi(1+G)|=0$. Third, $G \in O M^{*}$ on $[a, b]$ only if $(1) \Pi^{y}(1+G)$ 
exists for $a \leq x<y \leq b$, and (2) if $\epsilon>0$, then there exists a subdivision $D$ of $[a, b]$ such that, if $\left\{x_{i}\right\}_{i \neq 0}^{n}$ is a refinement of $D$ and $0 \leq p<q \leq n$, then

$$
\left|x_{p} \prod^{x} q(1+G)-\prod_{i=p+1}^{q}\left(1+G_{i}\right)\right|<\epsilon .
$$

We now state the main results of this paper.

Theorem 1. If $F$ and $G$ are functions from $R \times R$ to $N, F$ is in $O P^{\circ}$ and $S_{1} \cap S_{2}$ on $[a, b]$ and $G$ is in $O B^{\circ}$ and $S_{2}$ on $[a, b]$, then any two of the following statements imply the other:

(1) $F+G \in O M^{*}$ on $[a, b]$,

(2) $F \in O M^{*}$ on $[a, b]$, and

(3) $G \in O M^{*}$ on $[a, b]$.

Theorem 2. If $F$ and $G$ are functions from $R \times R$ to $N, F$ is in $O P^{\circ}$ and $S_{1} \cap S_{2}$ on $[a, b]$ and $G$ is in $O B^{\circ}$ and $S_{2}$ on $[a, b]$, then any two of the following statements imply the other:

(1) $F+G \in O M^{\circ}$ on $[a, b]$,

(2) $F \in O M^{\circ}$ on $[a, b]$, and

(3) $G \in O M^{\circ}$ on $[a, b]$.

Theorems 1 and 2 are not the same. A function can belong to $O M^{*}$ on $[a, b]$ without belonging to $O M^{\circ}$ on $[a, b]$. For example, if $G \in O B^{\circ}$ on $[a, b]$ and $\Pi^{y}(1+G)$ exists for $a \leq x<y \leq b$, then $G \in O M^{*}$ on $[a, b][7$, Theorem 1]; but, it is possible to construct a function $G$ such that $G \in O B^{\circ}$ on $[a, b]$, $\Pi^{y}(1+G)$ exists for $a \leq x<y \leq b$ and $G \notin O M^{\circ}$ on $[a, b]$ [4, pp. 153-154]. However, if $G$ is in $O M^{\circ}$ and $O P^{\circ}$ on $[a, b]$, then $G \in O M^{*}$ on $[a, b]$.

Theorem 2 is proved for functions from $R \times R$ to $R$ in a previous paper by the author [6, Theorem 1, p. 101]. However, that proof relies heavily on the commutativity of $R$ and thus is not the same as the proof presented in this paper. In this presentation, the lack of commutativity is handled by using a series representation for products.

The classes $O M^{*}$ and $O M^{\circ}$ are not as restricted as may initially appear. As noted before, if $G \in O B^{\circ}$ on $[a, b]$ and $\Pi^{y}(1+G)$ exists for $a \leq x<y$ $\leq b$, then $G \in O M^{*}$ on $[a, b][7$, Theorem 1]. For another example, suppose

$$
F(x, y)=\left[\begin{array}{cc}
0 & 0 \\
h(y)-b(x) & 0
\end{array}\right]
$$

for $a \leq x<y \leq b$, where $b$ is a quasi-continuous function from $R$ to $N$. Then, with a suitable norm, $F$ is in $O P^{\circ}, O M^{\circ}$ and $S_{1} \cap S_{2}$ on $[a, b]$. Thus, $F$ 
satisfies the hypotheses of Theorems 1 and 2; however, it does not necessarially follow that $F \in O B^{\circ}$ on $[a, b]$. With Theorems 1 and 2 and functions such as $F$, it is possible to construct many functions in $O M^{*}$ and $O M^{\circ} . A$ fundamental correspondence exists between sum and product integrals. In particular, if $G \in O B^{\circ}$ on $[a, b]$, then $\int_{a}^{b} G$ exists if and only if $\Pi_{x}^{y}(1+G)$ exists for $a \leq x<y \leq b\left[7\right.$, Theorem 4], and $G \in O A^{\circ}$ on $[a, b]$ if and only if $G \in O M^{\circ}$ on $[a, b][2$, Theorem 3.4, p. 301]. If $G$ is a function from $R \times R$ to $R$, then $G \in O A^{\circ}$ on $[a, b][8, \mathrm{p} .669]$. Further, there exist other systems such that the existence of $\int_{a}^{b} G$ is sufficient to imply that $G \in O A^{\circ}$ on $[a, b]$ [1, Theorem 2, p. 155], [2, Theorem 4.1, p. 304]. Thus, with the preceding results, many functions in $O M^{*}$ and $O M^{\circ}$ can be obtained. In addition, if $H \in O L^{\circ}$ on $[a, b]$ and $G \in O B^{\circ}$ on $[a, b]$, then $\Pi_{x}^{y}(1+H G)$ exists for $a \leq$ $x<y \leq b$ if $\Pi^{y}(1+G)$ exists for $a \leq x<y \leq b[7$, Theorem 5], and $H G \epsilon$ $O M^{\circ}$ on $[a, b]$ if $G \in O M^{\circ}$ on $[a, b][3$, Theorem 2, p. 494]. Therefore, there exist many functions to which the theorems of this paper apply.

We now establish Theorem 1. Several lemmas are needed.

Lemma 1. If $H$ and $G$ are functions from $R \times R$ to $N, H \in O L^{\circ}$ on $[a, b]$, $G \in O B^{\circ}$ on $[a, b]$ and either $\int_{a}^{b} G$ exists or $\Pi^{y}(1+G)$ exists for $a \leq x<$ $y \leq b$, then $\int_{a}^{b} H G$ and $\int_{a}^{b} G H$ exist and $\Pi_{x}^{y^{x}}(1+H G)$ and $\Pi_{x}^{y}(1+G H)$ exist for $a \leq x<y \leq b[7$, Theorem 5$]$.

Lemma 2. If $f$ is a function from $R$ to $R$ such that $(L R) \int_{a}^{b}(-d f) f^{n-i} f^{i}$ exists for $i=0,1, \ldots, n$, then

$$
\sum_{i=0}^{n}(L R) \int_{a}^{b}(-d f) f^{n-i} f^{i}=f^{n+1}(a)-f^{n+1}(b) .
$$

Proof. This result follows by applying the identity

$$
(r-s) \sum_{i=0}^{n} r^{n-i} s^{i}=r^{n+1}-s^{n+1}
$$

to the approximating sums of the integrals involved.

Lemma 3. If $\left\{F_{i}\right\}_{i=m}^{n}$ and $\left\{G_{i}\right\}_{i=m}^{n}$ are sequences of elements of $N$, then

$$
\prod_{i=m}^{n}\left(1+F_{i}+G_{i}\right)=\sum_{i=0}^{n+1-m} S_{i m n},
$$

where

$$
S_{0 p n}= \begin{cases}\Pi_{j=p}^{n}\left(1+F_{j}\right) & \text { if } 0<p \leq n, \\ 1 & \text { if } p>n,\end{cases}
$$


and

$$
S_{i p n}= \begin{cases}\sum_{j=p}^{n}\left[\Pi_{k=p}^{j-1}\left(1+F_{k}\right)\right] G_{j} S_{i-1, j+1, n} & \text { if } 0<p \leq n, \\ 0 & \text { if } p>n\end{cases}
$$

for $i=1,2, \ldots$.

Proof. This lemma can be established by induction.

Lemma 4. If $F$ and $G$ are functions from $R \times R$ to $N, F \in O P^{\circ}$ on $[a, b]$ and $G \in O B^{\circ}$ on $[a, b]$, then there exist a subdivision $D$ of $[a, b]$, a number $B$ and $a$ positive nondecreasing function $g$ defined on $[a, b]$ such that, if $\left\{x_{i}\right\}_{i=0}^{n}$ is a refinement of $D, j$ is a nonnegative integer and $0<p \leq q \leq n$, then

$$
\left|S_{j p q}\right| \leq B^{j+1}\left[g\left(x_{q}\right)-g\left(x_{p-1}\right)\right]^{j} / j !,
$$

where $S_{j p q}$ is defined in Lemma 3.

Proof. Since $F \in O P^{\circ}$ on $[a, b]$ and $G \in O B^{\circ}$ on $[a, b]$, there exist a subdivision $D$ of $[a, b]$ and a number $B$ such that, if $\left\{x_{i}\right\}_{i=0}^{n}$ is a refinement of $D$, then

(1) $\left|\Pi_{i=p+1}^{q}\left(1+F_{i}\right)\right|<B$ for $0 \leq p<q \leq n$, and

(2) $\sum_{i=1}^{n}\left|G_{i}\right|<B$.

Let $g$ be the function defined on $[a, b]$ such that

(1) $g(a)=1$, and

(2) $g(x)=1+\operatorname{lub}\left\{\Sigma_{J}|G|: J\right.$ a refinement of $\left.\left\{x_{i}\right\}_{i=0}^{p-1} \cup\{x\}\right\}$, where $0<$ $p \leq n$ and $x_{p-1}<x \leq x_{p}$.

Thus, $g$ is a positive nondecreasing function.

We use induction to establish the desired inequality. If $\left\{x_{i}\right\}_{i=0}^{n}$ is a refinement of $D$ and $0<p \leq q \leq n$, then

$$
\left|S_{0 p q}\right|=\left|\prod_{i=p}^{q}\left(1+F_{i}\right)\right| \leq B .
$$

Thus, the inequality is true for $j=0$.

Suppose the inequality holds for the nonnegative integer $j$. That is, if $\left\{x_{i}\right\}_{i=0}^{n}$ is a refinement of $D$ and $0<p \leq q \leq n$, then $(\dagger)$ holds.

We now establish that the inequality also holds for $j+1$. Suppose $\left\{x_{i}\right\}_{i=0}^{n}$ is a refinement of $D$ and $0<p \leq q \leq n$. To simplify notation in the following manipulations, let

$$
f(v)=g\left(x_{q}\right)-g(v)
$$

for $x_{p} \leq v \leq x_{q}$. Now, 


$$
\begin{aligned}
& \left|S_{j+1, p, q}\right|=\left|\sum_{i=p}^{q}\left[\prod_{k=p}^{i-1}\left(1+F_{k}\right)\right] G_{i} S_{j, i+1, q}\right| \\
& \leq B \sum_{i=p}^{q}\left|G_{i}\right|\left|S_{j, i+1, q}\right| \\
& \leq B \sum_{i=p}^{q}\left\{g\left(x_{i}\right)-g\left(x_{i-1}\right)\right\}\left\{B^{j+1}\left[g\left(x_{q}\right)-g\left(x_{i}\right)\right]^{i} / j !\right\} \\
& \leq B\left[(R) \int_{x_{p-1}}^{x_{q}} d g\left\{B^{j+1}\left[g\left(x_{q}\right)-g(v)\right]^{j} / j !\right\}\right] \\
& =\left[B^{j+2} / j !\right]\left[(R) \int_{x_{p-1}}^{x}(-d f) f^{j}\right] \\
& \leq\left[B^{j+2} /(j+1) !\right] \sum_{k=0}^{j}(L R) \int_{x_{p-1}}^{x_{q}}(-d f) f^{j-k} f^{k} \\
& =\left[B^{j+2} /(j+1) !\right]\left[f^{j+1}\left(x_{p-1}\right)-f^{j+1}\left(x_{q}\right)\right] \\
& =B^{j+2}\left[g\left(x_{q}\right)-g\left(x_{p-1}\right)\right]^{j+1} /(j+1) ! .
\end{aligned}
$$

Thus, the inequality holds for $j+1$. Hence, the inequality is valid for $j=$ $0,1,2, \ldots$ Therefore, Lemma 4 is established.

Lemma 5. If $F$ and $G$ are functions from $R \times R$ to $N, F \in O P^{\circ}$ on $[a, b]$. and $G \in O B^{\circ}$ on $[a, b]$, then $F+G \in O P^{\circ}$ on $[a, b]$.

Proof. This lemma follows as a corollary to Lemmas 3 and 4.

Lemma 5 is established in a previous paper by the author for functions from $R \times R$ to $R$ [5, Theorem $1(1 \rightarrow 2)$, p. 378]. However, the proof presented there is different from the proof employed in this paper.

Lemma 6. If $\left\{F_{i}\right\}_{i=m}^{n}$ and $\left\{G_{i}\right\}_{i=m}^{n}$ are sequences of elements of $N$, then

$$
\begin{aligned}
\prod_{i=m}^{n}\left(1+F_{i}+G_{i}\right)= & \prod_{i=m}^{n}\left(1+F_{i}\right) \\
& +\sum_{i=m}^{n}\left[\prod_{j=m}^{i-1}\left(1+F_{j}\right)\right] G_{i}\left[\prod_{j=i+1}^{n}\left(1+F_{j}+G_{j}\right)\right] .
\end{aligned}
$$

Proof. This lemma can be established by induction.

Lemma 7. If $G$ is a function from $R \times R$ to $N$ and $G \in O B^{\circ}$ on $[a, b]$, then the following statements are equivalent: 
(1) $\int_{a}^{b} G$ exists, and

(2) $\Pi_{x}^{y}(1+G)$ exists for $a \leq x<y \leq b$.

Further, if $G \in O B^{\circ}$ on $[a, b]$ and (1) or (2) is true, then $G \in O M^{*}$ on $[a, b]$.

Proof. If $G \in O B^{\circ}$ on $[a, b]$ and $\Pi^{y}(1+G)$ exists for $a \leq x<y \leq b$, then $G \in O M^{*}$ on $[a, b]\left[7\right.$, Theorem 1]. Also, if $G \in O B^{\circ}$ on $[a, b]$, then $\int_{a}^{b} G$ exists if and only if $\prod^{y}(1+G)$ exists for $a \leq x<y \leq b[7$, Theorem 4]. Thus, the lemma follows.

We now establish Theorem 1.

Proof of Theorem $1[(2),(3) \rightarrow(1)]$. We initially establish that $\sum_{i=0}^{\infty} P_{i}(x, y)$ converges uniformly and absolutely for $a \leq x<y \leq b$, where

$$
P_{0}(x, y)={ }_{x} \Pi^{y}(1+F)
$$

and

$$
P_{i}(x, y)=(L R) \int_{x}^{y} \prod^{u}(1+F) G P_{i-1}(v, y)
$$

for $a \leq x<y \leq b$ and $i=1,2, \ldots$. The existence of these integrals follows by applying Lemma 1 .

From Lemma 4 , there exist a subdivision $D_{1}$ of $[a, b]$, a number $B$ and a positive nondecreasing function $g$ defined on $[a, b]$ such that, if $\left\{x_{i}\right\}_{i=0}^{n}$ is a refinement of $D_{1}, i$ is a nonnegative integer and $0<p \leq q \leq n$, then

$$
\left|S_{i p q}\right| \leq B^{i+1}\left[g\left(x_{q}\right)-g\left(x_{p-1}\right)\right]^{i} / i !
$$

and

$$
\left|-S_{i p q}\right| \leq B^{i+1}\left[g\left(x_{q}\right)-g\left(x_{p-1}\right)\right]^{i} / i !,
$$

where $S_{i p q}$ is defined as in Lemma 3.

It follows from the result stated in the preceding paragraph that

$$
\left|P_{i}(x, y)\right| \leq B^{i+1}[g(y)-g(x)]^{i} / i !
$$

for $a \leq x<y \leq b$ and $i=0,1,2, \ldots$. Therefore, $\sum_{i=0}^{\infty} P_{i}$ converges uniformly and absolutely on $[a, b]$.

Suppose $a \leq x<y \leq b$. We now establish that ${ }_{x} \Pi^{y}(1+F+G)$ exists and is $\sum_{i=0}^{\infty} P_{i}(x, y)$. Let $\epsilon>0$.

There exists a positive integer $N$ such that

$$
\sum_{i=N+1}^{\infty} B^{i+1}[g(b)-g(a)]^{i} / i !<\epsilon / 3 .
$$

Further, from the existence properties of the integrals involved, there exists a subdivision $D_{2}$ of $[a, b]$ such that, if $\left\{x_{i}\right\}_{i=0}^{n}$ is a refinement of $D_{2}$ and $0 \leq p<q \leq n$, then 


$$
\left|\sum_{i=0}^{N} P_{i}\left(x_{p}, x_{q}\right)-\sum_{i=0}^{N} S_{i, p+1, q}\right|<\frac{\epsilon}{3} .
$$

Let $D$ denote a subdivision of $[x, y]$ which refines the intersection of $[x, y]$ and $D_{1} \cup D_{2}$ and has at least $N+1$ elements. Suppose $\left\{x_{i}\right\}_{i=0}^{n}$ is a refinement of $D$. Now,

$$
\begin{aligned}
\left|\sum_{i=0}^{\infty} P_{i}(x, y)-\prod_{i=1}^{n}\left(1+F_{i}+G_{i}\right)\right| \\
\quad=\left|\sum_{i=0}^{\infty} P_{i}(x, y)-\sum_{i=0}^{n} S_{i 1 n}\right| \\
\quad \leq\left|\sum_{i=0}^{N} P_{i}(x, y)-\sum_{i=0}^{N} S_{i 1 n}\right|+\left|\sum_{i=N+1}^{\infty} P_{i}(x, y)\right|+\left|-\sum_{i=N+1}^{n} S_{i 1 n}\right| \\
\leq \epsilon / 3+\epsilon / 3+\epsilon / 3=\epsilon .
\end{aligned}
$$

Hence, $\Pi^{y}(1+F+G)$ exists and is $\sum_{i=0}^{\infty} P_{i}(x, y)$.

We now establish that $F+G \in O M^{*}$ on $[a, b]$. Since $\Pi^{y}(1+F+G)$ exists for $a \leq x<y \leq b$, it is only necessary to establish the approximation part of the definition. Let $\epsilon>0$. Further, let $D_{1}, D_{2}$ and $N$ be defined as before.

Since $F$ is in $O M^{*}, O P^{\circ}$ and $S_{2}$ on $[a, b]$ and $G$ is in $O B^{\circ}$ and $S_{2}$ on $[a, b]$, there exists a subdivision $D_{3}$ of $[a, b]$ such that, if $\left\{x_{i}\right\}_{i=0}^{n}$ is a refinement of $[a, b], 0 \leq p<q \leq n$ and $q-p \leq N$, then

$$
\left|\prod_{p} \prod^{x}(1+F+G)-\prod_{i=p+1}^{q}\left(1+F_{i}+G_{i}\right)\right|<\epsilon .
$$

Let $D$ denote the subdivision $D_{1} \cup D_{2} \cup D_{3}$ of $[a, b]$. Suppose $\left\{x_{i}\right\}_{i=0}^{n}$ is a refinement of $D$ and $0 \leq p<q \leq n$. If $q-p \leq N$, then the desired inequality follows immediately from the definition of $D_{3}$. If $q-p \geq N$, then

$$
\begin{aligned}
\left|x_{p} \prod^{x}(1+F+G)-\prod_{i=p+1}^{q}\left(1+F_{i}+G_{i}\right)\right| \\
=\left|\sum_{i=0}^{\infty} P_{i}\left(x_{p}, x_{q}\right)-\sum_{i=0}^{p-q} S_{i, p+1, q}\right| \\
\leq\left|\sum_{i=0}^{N} P_{i}\left(x_{p}, x_{q}\right)-\sum_{i=0}^{N} S_{i, p+1, q}\right| \\
\quad+\left|\sum_{i=N+1}^{\infty} P_{i}\left(x_{p}, x_{q}\right)\right|+\left|-\sum_{i=N+1}^{p-q} s_{i, p+1, q}\right| \\
<\epsilon / 3+\epsilon / 3+\epsilon / 3=\epsilon .
\end{aligned}
$$


Therefore, $F+G \in O M^{*}$ on $[a, b]$. Thus, (2) and (3) imply (1).

Proof of Theorem $1[(1),(2) \rightarrow(3)]$. Since $F$ and $F+G$ are in $O M^{*}$ and $O P^{\circ}$ on $[a, b]$, the existence of

$$
\text { (LR) } \int_{x}^{y} \prod^{u}(1+F) G \prod_{\nu}^{y}(1+F+G)
$$

for $a \leq x<y \leq b$ can be established by employing Lemma 6 .

Since $F$ and $F+G$ are in $S_{1}$ on $[a, b]$, there exists a subdivision $\left\{x_{i}\right\}_{i=0}^{n}$ of $[a, b]$ such that, if $1 \leq i \leq n$ and $x_{i-1}<x<y<x_{i}$, then

$$
\left|\Pi^{y}(1+F)\right|<\frac{1}{2} \text { and }\left|\Pi^{y}(1+F+G)\right|<\frac{1}{2} \text {. }
$$

Suppose $1 \leq i \leq n$ and $x_{i-1}<x<y<x_{i}$. Let $J$ and $K$ represent interval functions such that, if $x \leq u<v \leq y$, then

$$
J(u, v)=\prod_{x}^{u}(1+F) \text { and } K(u, v)=\prod^{y}(1+F+G) .
$$

Since $J$ and $K$ are in $O L^{\circ}$ on $[a, b]$, it follows that $J^{-1}$ and $K^{-1}$ are also in $O L^{\circ}$ on $[a, b]$. Thus, from Lemma 1 and the existence of the integral in the preceding paragraph, we have that $\int_{x}^{y} G$ exists.

We have now established that, if $1 \leq i \leq n$ and $x_{i-1}<x<y<x_{i}$, then $\int_{x}^{y} G$ exists. From this and the fact that $G$ is in $O B^{\circ}$ and $S_{2}$ on $[a, b]$, it follows that $\int_{a}^{b} G$ exists. Hence, $G \in O M^{*}$ on $[a, b]$ by Lemma 7. Thus, (1) and (2) imply (3).

Proof of Theorem $1[(1),(3) \rightarrow(2)]$. It follows from Lemma 5 that $F+G$ $\epsilon O P^{\circ}$ on $[a, b]$. Further, $-G \in O M^{*}$ by Lemma 7. We have already established that (2) and (3) imply (1). Now, since $F+G-G \equiv F$, it follows that $F \in O M^{*}$ on $[a, b]$. Thus, (1) and (3) imply (2).

The proof of Theorem 1 is now complete. We next establish Theorem 2. One additional lemma is needed.

Lemma 8. If $G$ is a function from $R \times R$ to $N$ and $G \in O B^{\circ}$ on $[a, b]$, then the following statements are equivalent:

(1) $G \in O A^{\circ}$ on $[a, b]$, and

(2) $G \in O M^{\circ}$ on $[a, b][2$, Theorem $3.4, p .301]$.

Proof of Theorem $2[(2),(3) \rightarrow(1)]$. Since $F$ and $G$ are in $O P^{\circ}$ and $O M^{\circ}$ on $[a, b], F$ and $G$ are also in $O M^{*}$ on $[a, b]$. Hence, it follows from Theorem $1[(2),(3) \rightarrow(1)]$ that $\Pi_{x} \Pi^{y}(1+F+G)$ exists for $a \leq x<y \leq b$. Thus, it is only necessary to show that $\int_{a}^{b}|1+F+G-\Pi(1+F+G)|$ exists and is zero in order to establish that $F+G \in O M^{\circ}$ on $[a, b]$. Let $\epsilon>0$.

Since $F \in O M^{\circ}$ on $[a, b]$, there exists a subdivision $D_{1}$ of $[a, b]$ such that, if $\left\{x_{i}\right\}_{i=0}^{n}$ is a refinement of $D_{1}$, then 


$$
\sum_{i=1}^{n}\left|1+F_{i}-x_{i-1} \prod^{x_{i}}(1+F)\right|<\frac{\epsilon}{2} \text {. }
$$

We know that $F$ is in $O P^{\circ}$ and $O M^{*}$ on $[a, b]$. Further, $F+G \in O P^{\circ}$ on $[a, b]$ by Lemma 5 and $F+G \in O M^{*}$ on $[a, b]$ by Theorem $1[(2),(3) \rightarrow(1)]$. Now, since $G \in O B^{\circ}$ on $[a, b]$, it follows by using Lemma 6 that

$$
\text { (LR) } \int_{x}^{y} \prod^{u}(1+F) G \prod_{\nu}^{y}(1+F+G)
$$

exists and is

$$
\prod_{x}^{y}(1+F+G)-\prod_{x}^{y}(1+F)
$$

for $a \leq x<y \leq b$.

Since $F$ and $F+G$ are in $S_{1}$ and $O M^{*}$ on $[a, b]$, for each positive number $\beta$ there exists a subdivision $\left\{x_{i}\right\}_{i=0}^{n}$ of $[a, b]$ such that, if $1 \leq i \leq n$ and $x_{i-1}<x<y<x_{i}$, then

$$
\left|1-\prod_{x}^{y}(1+F)\right|<\beta \text { and }\left|1-\prod_{x}^{y}(1+F+G)\right|<\beta \text {. }
$$

By Lemma $8, G \in O A^{\circ}$ on $[a, b]$. Further, $F$ and $F+G$ are in $O P^{\circ}$ on $[a, b]$ and $G \in O B^{\circ}$ on $[a, b]$. From these facts, it follows that

$$
\int_{a}^{b}\left|G(x, y)-(L R) \int_{x}^{y} \prod_{x}^{u}(1+F) G \prod^{y}(1+F+G)\right|
$$

exists and is zero. Thus, there exists a subdivision $D_{2}$ of $[a, b]$ such that, if $\left\{x_{i}\right\}_{i=0}^{n}$ is a refinement of $D_{2}$, then

$$
\sum_{i=1}^{n}\left|G_{i}-(L R) \int_{x_{i-1}}^{x_{i}} x_{i-1} \prod^{u}(1+F) G \prod^{x_{i}}(1+F+G)\right|<\frac{\varepsilon}{2} .
$$

Let $D$ denote the subdivision $D_{1} \cup D_{2}$ of $[a, b]$. Suppose $\left\{x_{i}\right\}_{i=0}^{n}$ is a refinement of $D$. Now,

$$
\begin{aligned}
& \sum_{i=1}^{n}\left|1+F_{i}+G_{i}-x_{i-1} \prod^{x_{i}}(1+F+G)\right| \\
& =\sum_{i=1}^{n} \mid 1+F_{i}+G_{i}-\left[\prod_{i-1} \prod^{x_{i}}(1+F)\right. \\
& \left.\quad+(L R) \int_{x_{i-1} x_{i-1}}^{x_{i}} \prod^{u}(1+F) G \prod^{x_{i}}(1+F+G)\right] \mid \\
& \leq \sum_{i=1}^{n} \mid 1+F_{i}-x_{i-1} \prod^{x_{i}(1+F) \mid} \\
& \quad+\sum_{i=1}^{n}\left|G_{i}-(L R) \int_{x_{i-1} x_{i-1}}^{x_{i}} \prod^{u}(1+F) G \prod^{x_{i}}(1+F+G)\right| \\
& <\epsilon / 2+\epsilon / 2=\epsilon_{0}
\end{aligned}
$$


Therefore, $F+G \in O M^{\circ}$ on $[a, b]$. Thus, (2) and (3) imply (1).

Proof of Theorem $2[(1),(2) \rightarrow(3)]$. The proof of Theorem $2[(1),(2) \rightarrow$ (3)] is similar to the proof of Theorem $1[(1),(2) \rightarrow(3)]$. The only difference is that it is necessary to use Lemma 8 rather than Lemma 7. Thus, (1) and (2) imply (3).

Proof of Theorem $2[(1),(3) \rightarrow(2)]$. The proof of Theorem $2[(1),(3) \rightarrow$ (2)] is similar to the proof of Theorem $1[(1),(3) \rightarrow(2)]$. As before, the only difference is that it is necessary to use Lemma 8 rather than Lemma 7. Thus, (1) and (3) imply (2).

The proof of Theorem 2 is now complete.

\section{BIBLIOGRAPHY}

1. W. D. L. Appling, Interval functions and real Hilbert spaces, Rend. Circ. Mat. Palermo (2) 11 (1962), 154-156. MR 27 \#4040.

2. B. W. Helton, Integral equations and product integrals, Pacific J. Math. 16 (1966), 297-322. MR $32 \# 6167$.

3. - A product integral representation for a Gronwall inequality, Proc. Amer. Math. Soc. 23 (1969), 493-500. MR 40 \#1562.

4. J. C. Helton, An existence theorem for sum and product integrals, Proc. Amer. Math. Soc. 39 (1973), 149-154. 377-389.

5. - Bounds for products of interval functions, Pacific J. Math. 49 (1973), (1974), 96-103.

6. - Mutual existence of product integrals, Proc. Amer. Math. Soc. 42

7. - Mutual existence of sum and product integrals, Pacific J. Math. 56 (1975).

8. A. Kolmogoroff, Untersuchungen über den Integralbegriff, Math. Ann. 103 (1930), 654-696.

9. J. S. MacNerney, Integral equations and semigroups, Illinois J. Math. 7 (1963), 148-173. MR $26 \# 1726$.

10. P. R. Masani, Multiplicative Riemann integration in normed rings, Trans. Amer. Math. Soc. 61 (1947), 147-192. MR 8, 321.

DEPARTMENT OF MATHEMATICS, ARIZONA STATE UNIVERSITY, TEMPE, ARIZONA 